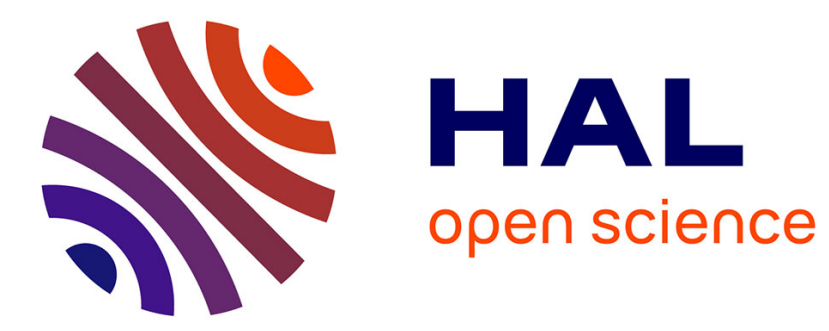

\title{
Stabilization of Chain of Integrators with Arbitrary Order in Finite-time
}

\author{
Konstantin Zimenko, Andrey Polyakov, Denis Efimov
}

\section{To cite this version:}

Konstantin Zimenko, Andrey Polyakov, Denis Efimov. Stabilization of Chain of Integrators with Arbitrary Order in Finite-time. 54th conference on Decision and Control 2015, IEEE, Dec 2015, Osaka, Japan. hal-01212600

\section{HAL Id: hal-01212600 \\ https://inria.hal.science/hal-01212600}

Submitted on 6 Oct 2015

HAL is a multi-disciplinary open access archive for the deposit and dissemination of scientific research documents, whether they are published or not. The documents may come from teaching and research institutions in France or abroad, or from public or private research centers.
L'archive ouverte pluridisciplinaire HAL, est destinée au dépôt et à la diffusion de documents scientifiques de niveau recherche, publiés ou non, émanant des établissements d'enseignement et de recherche français ou étrangers, des laboratoires publics ou privés. 


\title{
Stabilization of Chain of Integrators with Arbitrary Order in Finite-time*
}

\author{
Konstantin Zimenko ${ }^{1}$, Andrey Polyakov ${ }^{1,2}$, Denis Efimov ${ }^{1,2}$
}

\begin{abstract}
A control algorithm for finite-time stabilization of a chain of integrators with arbitrary order is introduced. The method is based on Implicit Lyapunov Function (ILF) approach with applying properties of homogeneous systems. Scheme of control parameter tuning is presented in Linear Matrix Inequality (LMI) form. The method is simple in implementation and does not assume any additional computational on-line procedures that is an improvement with respect to [8], [11]. The theoretical results are supported by numerical simulations.
\end{abstract}

\section{INTRODUCTION}

Frequently, strict quality requirements are imposed for design, operation and control of complex technical processes. One such requirement is completion of all transients in a finite time. In particular, this issue is relevant in control design for a variety of robotic and mechatronic devices, safety evaluation, aerospace applications, vehicles control systems, etc. Therefore the problems of finite-time control and finite-time observation are intensively studied for many years (see, for example, [1]-[4] and [5]-[7] correspondingly).

Solutions of the problem of control design for chain of integrators are quite sought-after. This is motivated by the fact that control scheme for chain of integrators can be easily extended to larger classes of systems (see, for example, [8]). Moreover, in many applications the nominal models have the form of chain of integrators, for instance, mechanical planar systems. There is a number of research results on the finite-time stabilization or regulation of a chain of integrators (see, for example, [9], [10]). However, most of them do not find an application in practice because of constructive and computational complexity. For instance, the methods mainly do not suggest constructive algorithms of parameter tuning and ensure only existence of suitable control parameters.

One of effective methods for the finite-time stability analysis is the method of Implicit Lyapunov Function (ILF) [8], [19], [20]. This method allows to check all stability conditions directly using implicitly defined Lyapunov function which is a solution of some algebraic equation. Note that for

\footnotetext{
*This work was supported by the Ministry of Education and Science of Russian Federation (Project 14.Z50.31.0031) and by Government of Russian Federation, Grant 074-U01.

${ }^{1}$ Konstantin Zimenko, Andrey Polyakov, Denis Efimov are with Department of Control Systems and Informatics, ITMO University, 49 Kronverkskiy av., 197101 Saint Petersburg, Russia. (e-mail: kostyazimenko@gmail.com).

${ }^{2}$ Andrey Polyakov, Denis Efimov are with Non-A INRIA - LNE, Parc Scientifique de la Haute Borne 40, avenue Halley Bat.A, Park Plaza 59650 Villeneuve d'Ascq (e-mail: denis.efimoveinria.fr, andrey.polyakove inria.fr). They are also with CRIStAL (UMR-CNRS 9189), Ecole Centrale de Lille, BP 48, Cite Scientifique, 59651 Villeneuve-d'Ascq, France.
}

such a stability analysis there is no need to solve this equation. Another issue that can be useful in analysis of finitetime stability is property of homogeneity. For instance, if an asymptotically stable system is homogeneous of negative degree, then it is finite-time stable.

The paper [11] is related to development of method for finite-time control of multiple integrators of arbitrary order together with implicit Lyapunov function of closed-loop system. Conditions of finite-time stability are formulated in the form of LMI that essentially simplifies the process of tuning of control parameters. However, the practical realization of the ILF control algorithm requires development of special computational procedure for on-line computation of the ILF value at the current state (the control law depends on ILF value explicitly).

The present paper addresses the problem of a control design for the finite-time stabilization of a chain of integrators with arbitrary order. The paper represents an extension and simplification of results in [11]. The developed finitetime control law does not depend on implicitly defined Lyapunov function that allows to avoid any additional online procedures. The result is obtained by using the method of Implicit Lyapunov Function with applying properties of homogeneous systems. As in [11] parameter tuning is presented in LMI form.

The paper is organized as follows. Notation used in the paper is given in the next section. Problem statement is considered in Section III. Some background notions about finitetime stability, ILF method and homogeneity are introduced in Section IV. Control design algorithm and some remarks about proposed control scheme are presented in Section V. Section VI contains simulation results for effectiveness demonstration of proposed control. Finally, concluding remarks and possible directions for future research are presented in Section VII.

\section{NOTATION}

Through the paper the following notation will be used:

- $\mathbb{R}_{+}=\{x \in \mathbb{R}: x>0\}$, where $\mathbb{R}$ is the set of real number;

- $\|\cdot\|$ is the Euclidian norm in $\mathbb{R}^{n}$, i.e. $\|x\|=$ $\sqrt{x_{1}^{2}+\cdots+x_{n}^{2}}$ for $x \in \mathbb{R}^{n}$

- the positive (negative) definiteness (semi-definiteness) of a symmetric matrix $P=P^{T} \in \mathbb{R}^{n \times n}$ is denoted by $P>0(P<0, P \geq 0, P \leq 0)$;

- for a symmetric matrix $P=P^{T}$ the minimal and maximal eigenvalues are denoted by $\lambda_{\min }(P)$ and $\lambda_{\max }(P)$, respectively; 
- a continuous function $\sigma: \mathbb{R}_{+} \cup\{0\} \rightarrow \mathbb{R}_{+} \cup\{0\}$ belongs to the class $\mathcal{K}$ if $\sigma(0)=0$ and the function is strictly increasing;

- $\operatorname{diag}\left\{\lambda_{i}\right\}_{i=1}^{n}$ is the diagonal matrix with the elements $\lambda_{i}$ on the main diagonal.

\section{PROBLEM STATEMENT}

Consider the control system

$$
\dot{x}=A x+b u,
$$

where $x \in \mathbb{R}^{n}$ is the state vector, $u \in \mathbb{R}$ is the control input,

$$
A=\left(\begin{array}{ccccc}
0 & 1 & 0 & \cdots & 0 \\
0 & 0 & 1 & \cdots & 0 \\
\vdots & \vdots & \vdots & \ddots & \vdots \\
0 & 0 & 0 & \cdots & 1 \\
0 & 0 & 0 & \cdots & 0
\end{array}\right) \quad \text { and } b=\left(\begin{array}{c}
0 \\
0 \\
\vdots \\
0 \\
1
\end{array}\right)
$$

that describes a chain of integrators.

The aim of the paper is to develop the finite-time stabilizing control algorithm to the system (1), which satisfies two restrictions:

- it has the form of the explicit full-state feedback and its application does not require additional on-line computations;

- it admits the simple computational scheme (e.g. LMIbased) scheme for parameters tuning.

\section{PRELIMINARIES}

\section{A. Finite-time Stability}

Consider the system of the form

$$
\dot{x}=f(t, x), \quad x(0)=x_{0},
$$

where $x \in \mathbb{R}^{n}$ is the state vector, $f \in \mathbb{R}_{+} \times \mathbb{R}^{n} \rightarrow$ $\mathbb{R}^{n}$ is a nonlinear continuous vector field, which can be discontinuous with respect to the state variable. In this case the solutions $x\left(t, x_{0}\right)$ of the system (2) are understood in the sense of Filippov [13].

According to [13] an absolutely continuous function $x\left(t, x_{0}\right)$ is called a solution of the Cauchy problem associated to (2) if $x\left(0, x_{0}\right)=x_{0}$ and for almost all $t>0$ it satisfies the following differential inclusion

$$
\dot{x} \in K[f](t, x)=c o \bigcap_{\varepsilon>0} \bigcap_{\mu(N)=0} f(t, B(x, \varepsilon) \backslash N),
$$

where $\operatorname{co}(M)$ defines the convex closure of the set $M$, $B(x, \varepsilon)$ is the ball with the center at $x \in \mathbb{R}^{n}$ and the radius $\varepsilon$, the equality $\mu(N)=0$ means that the measure of the set $x \in \mathbb{R}^{n}$ is zero.

Assume that the origin is an equilibrium of the system (2) and it has uniqueness of solutions in forward time.

Definition 1 ([1], [2], [14]):

The origin of system (2) is said to be globally finite-time stable if:

- Finite-time attractivity: there exists so-called settling time function $T: \mathbb{R}^{n} \backslash\{0\} \rightarrow \mathbb{R}_{+}$such that $\lim _{t \rightarrow T\left(x_{0}\right)} x\left(t, x_{0}\right)=0$ for all $x_{0} \in \mathbb{R}^{n} \backslash\{0\}$.
- Lyapunov stability: there exists a function $\delta \in \mathcal{K}$ such that $\left\|x\left(t, x_{0}\right)\right\| \leq \delta\left(\left\|x_{0}\right\|\right)$ for all $x_{0} \in \mathbb{R}^{n}$.

Notice that [11], [12]:

- finite-time stability is obtained through an "infinite eigenvalue assignation" for the system at the origin;

- the settling time function $T\left(x_{0}\right)$ evaluates the time for a solution to reach the equilibrium;

- the right hand side of the ordinary differential equation cannot be locally Lipschitz at the origin.

\section{B. Homogeneity}

Homogeneity [15]-[17] is an intrinsic property whereby objects such as functions or vector fields remain consistent with respect to some scaling operation called a dilation.

For fixed $r_{i} \in \mathbb{R}_{+}, i \in\{1, \ldots, n\}$ called weights and $\lambda>0$ one can define:

- the vector of weights $r=\left(r_{1}, \ldots, r_{n}\right)^{T}$;

- the dilation matrix

$$
D(\lambda)=\operatorname{diag}\left\{\lambda^{r_{i}}\right\}_{i=1}^{n}
$$

and correspondingly $D(\lambda) x=\left(\lambda^{r_{1}} x_{1}, \ldots, \lambda^{r_{n}} x_{n}\right)^{T}$ for $x=\left(x_{1}, \ldots, x_{n}\right)^{T} \in \mathbb{R}^{n}$.

Definition 2 [17]:

A function $g: \mathbb{R}^{n} \rightarrow \mathbb{R}$ (vector field $f: \mathbb{R}^{n} \rightarrow \mathbb{R}^{n}$ ) is said to be $r$-homogeneous of degree $m$ iff $g(D(\lambda) x)=\lambda^{m} g(x)$ $\left(f(D(\lambda) x)=\lambda^{m} D(\lambda) f(x)\right)$ for all $\lambda>0$ and $x \in \mathbb{R}^{n}$.

Let us introduce the homogeneous unit sphere

$$
S_{r}=\left\{x \in \mathbb{R}^{n}:\|x\|_{r}=1\right\},
$$

where $\|x\|_{r}$ represents the homogeneous norm of $x \in \mathbb{R}^{n}$ defined by

$$
\|x\|_{r}=\left(\sum_{i=1}^{n}\left|x_{i}\right|^{\frac{\rho}{r_{i}}}\right)^{\frac{1}{\rho}}
$$

for $\rho \geq \max _{i} r_{i}$.

Obviously, each homogeneous norm is $r$-homogeneous of degree 1 and positive definite. For any $x \in \mathbb{R}^{n}$ there are $y \in S_{r}$ and $\lambda=\|x\|_{r}$ such that $x=D(\lambda) y[18]$.

Theorem 1 [18]

Let $f$ be a $r$-homogeneous continuous vector field on $\mathbb{R}^{n}$ such that the system (2) is a locally asymptotically stable. Then it is globally asymptotically stable and there exists a continuously differentiable $r$-homogeneous Lyapunov function $V$ for (2).

Note that in accordance with [18] there exist constants $c_{1}, c_{2}>0$ such that

$$
c_{1}\|x\|_{r}^{m} \leq V(x) \leq c_{2}\|x\|_{r}^{m},
$$

where $m$ is degree of $r$-homogeneous Lyapunov function.

Theorem 2 [18]

Let $f: \mathbb{R}^{n} \rightarrow \mathbb{R}^{n}$ be defined on $\mathbb{R}^{n}$ and be a continuous $r$-homogeneous vector field with a negative degree. If the origin of the system (2) is locally attractive then it is globally finite time stable. 


\section{Implicit Lyapunov Function Method}

The theorem given below presents the ILF method for finite-time stability analysis.

Theorem 3 [8], [12]

If there exists a continuous function

$$
\begin{aligned}
& Q: \mathbb{R}_{+} \times \mathbb{R}^{n} \rightarrow \mathbb{R} \\
& (V, x) \mapsto Q(V, x)
\end{aligned}
$$

such that

C1) $Q(V, x)$ is continuously differentiable for $\forall x \in$ $\mathbb{R}^{n} \backslash\{0\}$ and $\forall V \in \mathbb{R}_{+}$;

$C 2$ ) for any $x \in \mathbb{R}^{n} \backslash\{0\}$ there exist $V^{-} \in \mathbb{R}_{+}$and $V^{+} \in$ $\mathbb{R}_{+}$:

$$
Q\left(V^{-}, x\right)<0<Q\left(V^{+}, x\right)
$$

C3) for $\Omega=\left\{(V, x) \in \mathbb{R}^{n+1}: Q(V, x)=0\right\}$

$$
\lim _{\substack{x \rightarrow 0 \\(V, x) \in \Omega}} V=0^{+}, \lim _{\substack{V \rightarrow 0^{+} \\(V, x) \in \Omega}}\|x\|=0, \lim _{\substack{\|x\| \rightarrow \infty \\(V, x) \in \Omega}} V=+\infty ;
$$

C4) the inequality

$$
-\infty<\frac{\partial Q(V, x)}{\partial V}<0
$$

holds for $\forall V \in \mathbb{R}_{+}$and $\forall x \in \mathbb{R}^{n} \backslash\{0\}$;

C5) the inequality

$$
\frac{\partial Q(V, x)}{\partial x} f(x) \leq \eta V^{1-\mu} \frac{\partial Q(V, x)}{\partial V}
$$

holds $\forall(V, x) \in \Omega$, where $0<\mu \leq 1$ and $\eta>0$ are some constants.

Then the origin of the system (2) is globally finite-time stable with the following settling time estimate

$$
T\left(x_{0}\right) \leq \frac{V_{0}^{\mu}}{\eta \mu} .
$$

\section{MAIN RESULT}

Introduce the ILF function

$$
Q(V, x):=x^{T} D\left(V^{-1}\right) P D\left(V^{-1}\right) x-1,
$$

where $P=P^{T} \in \mathbb{R}^{n \times n}$ is a symmetric positive definite matrix, $D(\lambda)$ is the dilation matrix of the form

$$
D(\lambda)=\operatorname{diag}\left\{\lambda^{1+(n-i) \mu}\right\}
$$

for $0<\mu \leq 1$.

Let us denote the diagonal matrices $H_{\mu}=\operatorname{diag}_{n}\{-1-(n-i) \mu\}_{i=1}^{n} \quad$ and $H(\lambda)=$ $\operatorname{diag}\left\{\lambda^{(n+1-i) \mu}\right\}_{i=1}^{n}$.

\section{Theorem 4}

If the following statements hold:

1) the system of matrix inequalities

$$
\left\{\begin{array}{l}
A X+X A^{T}+b y+y^{T} b^{T}+\alpha X+\beta I_{n} \leq 0 \\
X H_{\mu}+H_{\mu} X<0, \quad X>0, \quad \beta I_{n} \geq \gamma X \\
\left(\begin{array}{cc}
\gamma & y \\
y^{T} & X
\end{array}\right) \geq 0, \quad\left(\begin{array}{cc}
X & I_{n}-H(c) \\
I_{n}-H(c) & \beta I_{n}
\end{array}\right) \geq 0
\end{array}\right.
$$

is feasible for some $\mu \in(0,1], \alpha, \beta, \gamma, c \in \mathbb{R}_{+}: \alpha>$ $\beta, X \in \mathbb{R}^{n \times n}, y \in \mathbb{R}^{1 \times n}$;
2) there exists a $c_{u}$ that satisfies one of the two following inequalities:

$$
\frac{c_{1}}{c} \geq c_{u} \geq c_{2}
$$

or

$$
c_{1} \geq c_{u} \geq \frac{c_{2}}{c}
$$

where the coefficients $c_{1}$ and $c_{2}$ correspond to the inequality (5).

Then the control of the form

$$
u(x)=\left(c_{u}\|x\|_{r}\right)^{1-\mu} k D\left(\left(c_{u}\|x\|_{r}\right)^{-1}\right) x,
$$

where $k=y X^{-1}$ stabilizes the origin of the system (1) in a finite time.

Let us make some remarks about presented control scheme:

- For practical implementation of the control (11) feasibility of inequality (9) or (10) is required. Therefore, it is necessary to estimate the coefficients $c_{1}$ and $c_{2}$. In this case in accordance with (7) one can conclude that the Lyapunov function $V(x)$ is $r$-homogeneous of degree 1 as $Q(V, D(\lambda) x)=Q\left(\lambda^{-1} V, x\right)$, i.e. $V(D(\lambda) x)=\lambda V(x)$. Then rewrite (5)

$$
c_{1}\|x\|_{r} \leq V(x) \leq c_{2}\|x\|_{r} .
$$

The inequality (12) can be rewritten in the following form

$$
c_{1}\left\|D\left(V^{-1}\right) x\right\|_{r} \leq 1 \leq c_{2}\left\|D\left(V^{-1}\right) x\right\|_{r}
$$

Denote $z=\left(z_{1}, \ldots, z_{n}\right)^{T}:=D\left(V^{-1}\right) x$. Hence

$$
c_{1}^{\rho} \sum_{i=1}^{n}\left|z_{i}\right|^{\rho / r_{i}} \leq 1 \leq c_{2}^{\rho} \sum_{i=1}^{n}\left|z_{i}\right|^{\rho / r_{i}} .
$$

From the other side, $x^{T} D\left(V^{-1}\right) P D\left(V^{-1}\right) x=1$ implies

$$
\frac{1}{\lambda_{\max }(P)} \leq \sum_{i=1}^{n} z_{i}^{2} \leq \frac{1}{\lambda_{\min }(P)} .
$$

If $P \geq I_{n}$ (or $X \leq I_{n}$ that coincides with the fourth inequality of (8) for $\beta=\gamma$ ), then $\left\|z_{i}\right\| \leq 1$ and hence $\left|z_{i}\right| \leq 1$. For $\rho=1+(n-1) \mu$ using inequality of arithmetic and quadratic means one can obtain

$$
\sum_{i=1}^{n}\left|z_{i}\right|^{\rho / r_{i}} \leq \sum_{i=1}^{n}\left|z_{i}\right| \leq \sqrt{n}\|z\|
$$

and

$$
c_{1} \leq \frac{1}{\sqrt[2 \rho]{n}}
$$

On the other hand, if we choose $\mu \in\left(0, \frac{1}{n-1}\right]$ then $\frac{\rho}{r_{i}} \leq 2$ and $\left|z_{i}\right| \leq 1$ implies

$$
\left|z_{i}\right|^{2} \leq\left|z_{i}\right|^{\frac{\rho}{r_{i}}}
$$

Therefore,

$$
\sum_{i=1}^{n}\left|z_{i}\right|^{2} \leq \sum_{i=1}^{n}\left|z_{i}\right|^{\rho / r_{i}}
$$


and

$$
c_{2} \geq \sqrt[p]{\lambda_{\max }(P)} .
$$

Thus, one can rewrite the system (8)

$$
\left\{\begin{array}{l}
A X+X A^{T}+b y+y^{T} b^{T}+\alpha X+\beta I_{n} \leq 0 \\
X H_{\mu}+H_{\mu} X<0, \quad I_{n} \geq X>0, \\
\left(\begin{array}{cc}
\beta & y \\
y^{T} & X
\end{array}\right) \geq 0, \quad\left(\begin{array}{cc}
X & I_{n}-H(c) \\
I_{n}-H(c) & \beta I_{n}
\end{array}\right) \geq 0
\end{array}\right.
$$

and inequalities (9), (10) take the forms

$$
\frac{\sqrt[2 \rho]{n}}{c} \geq c_{u} \geq \sqrt[\rho]{\lambda_{\max }(P)}
$$

and

$$
\sqrt[2 \rho]{n} \geq c_{u} \geq \frac{\sqrt[\rho]{\lambda_{\max }(P)}}{c}
$$

correspondingly for $\mu \in\left(0, \frac{1}{n-1}\right], \rho=1+(n-1) \mu$.

- The advantage of proposed control scheme is based on simple tuning of control parameters presented in LMI. For any fixed $\alpha, \mu \in \mathbb{R}_{+}$the first three inequalities of the system (8) are feasible at least for sufficiently small $\mu \in(0,1]$ [11]. Moreover, the last three inequalities of the system (8) are defined by the inequality

$$
\begin{aligned}
& \beta^{2} P \geq \\
& \left(I_{n}-H\left(\frac{V}{c_{u}\|x\|_{r}}\right)\right) k^{T} k\left(I_{n}-H\left(\frac{V}{c_{u}\|x\|_{r}}\right)\right),
\end{aligned}
$$

which also becomes feasible for sufficiently small $\mu$, since $\left(I_{n}-H\left(\frac{V}{c_{u}\|x\|_{r}}\right)\right) \rightarrow 0$ for $\mu \rightarrow 0$. Thus, one can always find such $\mu$ for which the system (1) is finite-time stable with control in the form (11).

- If $c_{u}$ satisfies the inequality (9) then the control (11) is bounded with $|u(x)| \leq\left(c_{2}\|x\|_{r}\right)^{1-\mu} \sqrt{\gamma}$. Indeed,

$\frac{1}{\gamma} u^{2}(x)=$

$\frac{1}{\gamma}\left(c_{u}\|x\|_{r}\right)^{2-2 \mu} x^{T} D\left(\left(c_{u}\|x\|_{r}\right)^{-1}\right) k^{T} k D\left(\left(c_{u}\|x\|_{r}\right)^{-1}\right) x \leq$ $\frac{1}{\gamma} V^{2-2 \mu} x^{T} D\left(V^{-1}\right) k^{T} k D\left(V^{-1}\right) x \leq V^{2-2 \mu}=$ $V^{2-2 \mu} x^{T} D\left(V^{-1}\right) P D\left(V^{-1}\right) x \Rightarrow \frac{1}{\gamma} k^{T} k \leq P$

coincides with the matrix inequality $\left(\begin{array}{cc}\gamma & y \\ y^{T} & X\end{array}\right) \geq$ 0 in LMI form. As $V \leq c_{2}\|x\|_{r}$ then $u^{2}(x) \leq$ $\gamma\left(c_{2}\|x\|_{r}\right)^{2-2 \mu}$.

- In order to estimate the settling time function the following matrix inequality

$$
X H_{\mu}+H_{\mu} X \geq-\nu X
$$

can be added to (8) for some $\nu>0$. Then the settling time function estimate has the form

$$
T\left(x_{0}\right) \leq \frac{\nu V_{0}^{\mu}}{\mu(\alpha-\beta)} \leq \frac{\nu c_{2}\left\|x_{0}\right\|_{r}^{\mu}}{\mu(\alpha-\beta)} .
$$

- The system (1) is $r$-homogeneous of degree $-\mu$ with the vector of weights $r=(1+(n-1) \mu, 1+(n-2) \mu \ldots, 1)^{T}$. Indeed, since homogeneous norm is $r$-homogeneous of degree 1 , i.e. $\|D(\lambda) x\|_{r}=\lambda\|x\|_{r}$ :

$$
\begin{aligned}
& u(D(\lambda) x)= \\
& \left(c_{u}\|D(\lambda) x\|_{r}\right)^{1-\mu} k D\left(\left(c_{u}\|D(\lambda) x\|_{r}\right)^{-1}\right) D(\lambda) x= \\
& \lambda^{1-\mu}\|x\|_{r} k D\left(\lambda^{-1}\left(c_{u}\|x\|_{r}\right)^{-1}\right) D(\lambda) x=\lambda^{1-\mu} u(x)
\end{aligned}
$$

and $A D(\lambda) x+B u(D(\lambda) x)=\lambda^{-\mu} D(\lambda)(A x+B u(x))$.

- In comparison with the results of the work [11] the control (11) does not require any additional computational power for on-line computation of the ILF value at the current state. This issue significantly simplifies the practical realization of the developed finite-time control scheme.

\section{NUMERICAL EXAMPLE}

Consider the system (1) for $n=2$. Define the finite-time control $u$ in the form (11) with the parameter $\mu=0.5$, where the matrix $P \in \mathbb{R}^{2 \times 2}$ and the vector $k \in \mathbb{R}^{1 \times 2}$ are obtained from the system of matrix inequalities (8) with parameters $c=0.02, \alpha=0.1, \rho=1+(n-1) \mu=1.5$ and $\gamma=\beta=$ $3.8605 * 10^{-4}$ :

$$
\begin{aligned}
P & =\left(\begin{array}{cc}
55.9229 & 9.9147 \\
9.9147 & 242.5282
\end{array}\right), \\
k & =\left(\begin{array}{ll}
-0.2245 & -0.2515
\end{array}\right) .
\end{aligned}
$$

As $\gamma=\beta$ in accordance with (13) and (14) we can select $c_{1}=0.7937, c_{2}=38.9465$ and coefficient $c_{u}$ due to (16) should satisfy the following expression

$$
39.6850 \geq c_{u} \geq 38.9465 .
$$

The simulation results for $c_{u}=39$ and $x_{1}(0)=3$, $x_{2}(0)=1$ are presented in the Fig. 1, Fig. 2. The system states are shown in the Fig. 1. Fig. 2 presents the control signal $u$ with its bounds.

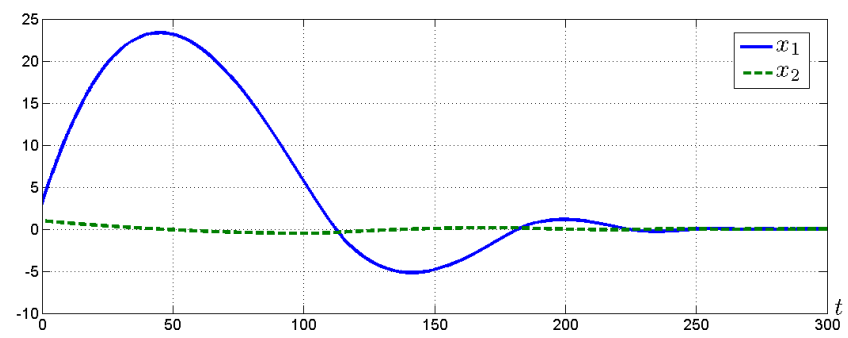

Fig. 1. Transients of the system states for the finite-time control

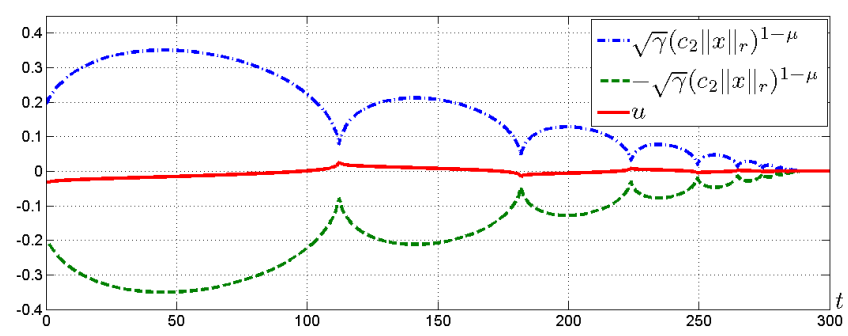

Fig. 2. The control signal $u$ with its bounds

The corresponding simulation results for control algorithm presented in [11] with the same values of $P, k, \mu, \alpha$ and $\beta$ are shown in the Fig. 3, Fig. 4. 


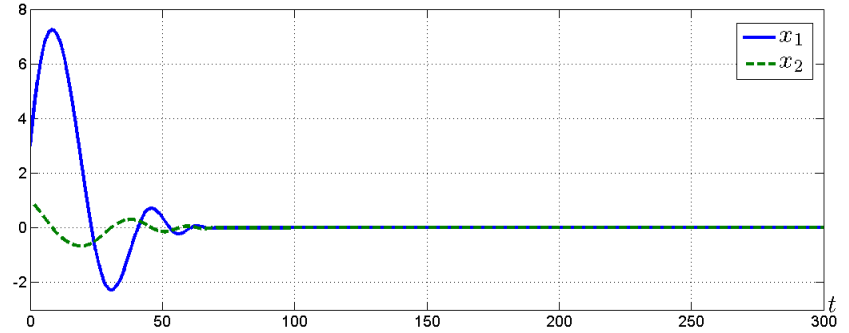

Fig. 3. Transients of the system states for the finite-time control presented in [11]

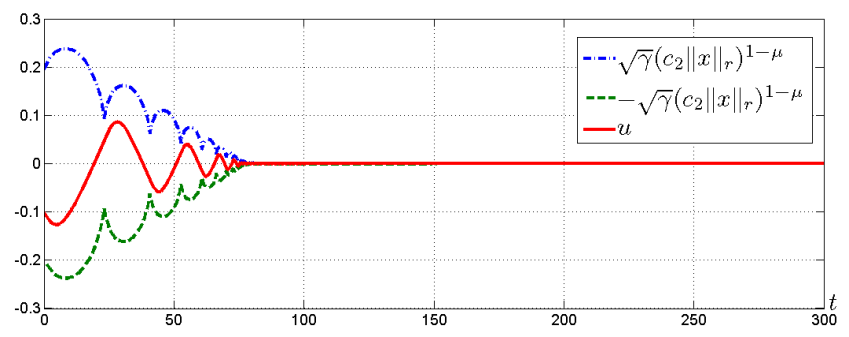

Fig. 4. The control signal $u$ with its bounds for the finite-time control presented in [11]

\section{CONCLUSIONS}

The paper presents control algorithm for finite-time stabilization of chain of integrators with arbitrary order. The control design procedure is based on application of the ILF method and some properties of homogeneous systems. Tuning of control parameters is presented in the form of linear matrix inequalities. Numerical example demonstrates the effectiveness of proposed control.

This work is an extension of the paper [11] and in comparison with it obtained control $u$ does not require online procedure for computation of the ILF value at the current state. This issue allows to simplify the practical realization of the control scheme.

There are many possible directions for further research. For instance, studying robustness with respect to delays or extension of the present result for MIMO and nonlinear systems.

\section{REFERENCES}

[1] S.P. Bhat, D.S. Bernstein, Finite-time stability of continuous autonomous systems, SIAM Journal of Control and Optimization, vol. 38(3), pp. 751-766, 2000.

[2] E. Roxin, On finite stability in control systems, Rendiconti del Circolo Matematico di Palermo, vol. 15(3), pp. 273-283, 1966.

[3] V. Haimo, Finite time controllers, SIAM Journal of Control and Optimization, vol. 24(4), pp. 760-770, 1986.

[4] E. Moulay and W. Perruquetti, Finite-time stability and stabilization: State of the art, Lecture Notes in Control and Information Sciences, vol. 334, pp. 23-41, 2006.

[5] R. Engel and G. Kreisselmeier, A continuous-time observer which converges in finite time, IEEE Transactions on Automatic Control, vol. 47, pp. 1202-1204, 2002.

[6] W. Perruquetti, T. Floquet, and E. Moulay, Finite-time observers: Application to secure communication, IEEE Transactions on Automatic Control, vol. 53(1), pp. 356-360, 2008.
[7] Y. Shen, Y. Huang, and J. Gu, Global finite-time observers for lipschitz nonlinear systems, IEEE Transactions on Automatic Control, vol. 56(2), pp. 418-424, 2011.

[8] A. Polyakov, D. Efimov, W. Perruquetti, Robust Stabilization of MIMO Systems in Finite/Fixed Time, Int. J. Robust. Nonlinear Control, DOI 10.1002/rnc.3297, 2015.

[9] A. Levant, Homogeneity approach to high-order sliding mode design, Automatica, vol. 41, pp. 823-830, 2005.

[10] E. Bernuau, W. Perruquetti, D. Efimov, E. Moulay, Finite-time output stabilization of the double integrator, in Proc. 51st IEEE Conference on Decision and Control, Maui, USA, pp. 5906-5911, 2012.

[11] A. Polyakov, D. Efimov, W. Perruquetti, Finite-time stabilization using implicit lyapunov function technique, in Proc. 9th Symposium on Nonlinear Control Systems, Toulouse, France, pp. 140-145, Sep. 2013

[12] A. Polyakov, D. Efimov, W. Perruquetti, Finite-time and fixed-time stabilization: Implicit Lyapunov function approach, Automatica, Volume 51, pp. 332-340, 2015.

[13] A. F. Filippov. Differential equations with discontinuous right-hand sides, Kluwer, Dordrecht, 1988.

[14] Y. Orlov, Finite time stability and robust control synthesis of uncertain switched systems, SIAM Journal of Control and Optimization, vol. 43(4), pp. 1253-1271, 2005.

[15] V. I. Zubov, Methods of A.M. Lyapunov and Their Applications, Noordhoff Ltd, Groningen, 263 p., 1964.

[16] H. Hermes, Nilpotent approximations of control systems and distributions, SIAM Journal of Control and Optimization, vol. 24, pp. 731736 , 1986.

[17] V. I. Zubov, On systems of ordinary differential equations with generalized homogenous right-hand sides, Izvestia vuzov. Mathematica, vol. 1, pp. 8088, 1958 (in Russian).

[18] A. Bacciotti, L. Rosier, Lyapunov Functions and Stability in Control Theory, Springer, 237 p., 2005.

[19] J. Adamy, A. Flemming, Soft variable-structure controls: a survey, Automatica, vol. 40, pp. 1821-1844, 2004.

[20] V. I. Korobov, A general approach to synthesis problem, Doklady Academii Nauk SSSR, vol. 248, pp. 1051-1063, 1979. 